\title{
VOYAGES, MOBILITÉS, LUMIÈRES
}

\author{
Daniel RochE
}

RÉSUMÉ : Depuis longtemps et aujourd'hui encore, les récits de voyage fascinent un vaste public. Il est important de débattre de leur modèle et du modèle de construction intellectuelle et sensible qu'ils ont proposé. Lien prioritaire de l'acculturation des élites européennes, ils ne peuvent être séparés d'une circulation beaucoup plus vaste et qui intéresse toute la société. C'est par rapport aux principes de la morale sociale ancienne que le concept de mobilité permet de prendre en compte l'ensemble des pratiques sociales et de confronter circulation et localisation. Les catégories principales de la culture, espace, temps, sociabilité, contraintes et libertés sociales, tissent alors une manière de comprendre le changement.

MoTS-CLÉS : voyage, mobilité, Lumières, Europe.

ABSTRACT: For a very long time and still today, travel stories have fascinated a vast public. It is important to debate their role and the model for intellectual and sensitive construction they propose. An essential link for the European elite's acculturation, they cannot for as much be separated from a much wider circulation which concerns society as a whole. In relation to the social morals of our past, the concept of mobility allows us to appreciate the totality of social practices and to confront the notions of movement and localization. The main cultural categories of space, time, sociability, social constraints and freedoms, are thus creating a structure for our understanding of change.

KEYWORDS : travel, mobility, Enlightenment, Europe. 
ZUSAMMENFASSUNG : Reiseberichte faszinieren seit langem und haben bis heute ein breites Publikum. Wichtig ist es, über ihre Bedeutung und über das von ihnen angebotene intellektuelle und physische Konstruktionsmodell zu diskutieren. Als verbindender Hauptbestandteil der Akkulturation europäischer Eliten können sie jedoch nicht von einer umfassenderen Mobilität getrennt werden, die die ganze Gesellschaft betrifft. Im Bezug zur alten sozialen Ordnung wird es mit dem Begriff «Mobilität» möglich gemacht, alle sozialen Verhaltensweisen zu berücksichtigen und die Ortsfestigkeit der Beweglichkeit gegenüberzustellen. Die grundsätzlichen Kategorien Kultur, Raum, Zeit, Gesellschaft, soziale Freiheit und Verpflichtungen greifen also ineinander und lassen auf diese Weise den Wandel erklären.

STICHWÖRTER : Reise, Mobilität, Aufklärung, Europa.

Daniel Roche, né en 1935, est professeur au Collège de France, titulaire de la chaire d'histoire de la France des Lumières. Ses principaux ouvrages sont Le Siècle des Lumières en Province (1978); Le Peuple de Paris (1981); Journal de ma vie (1982); Les Français et l'Ancien Régime, avec Pierre Goubert (1984) ; Les Républicains des lettres. Gens de culture et Lumières au XVIII siècle (1988); La Culture des apparences (1989); La France des Lumières (1993) ; Histoire des choses banales (1997); La Ville promise (2000) ; Humeurs vagabondes. De la circulation des hommes et de l'utilité des voyages (2003).

Adresse : Collège de France, 11 place Marcelin-Berthelot, F-75231 Paris Cedex 05.

Courrier électronique : danielroche@collegedefrance.fr 
Le but de cette présentation est de préciser quelques-uns des principes qui ont guidé depuis une dizaine d'années ma réflexion sur la culture de la mobilité dans les sociétés traditionnelles, et dans l'espace européen. Le XVIII siècle y occupe, on s'en doute, une place particulière car il a toujours été considéré comme le moment privilégié d'un double phénomène. Celui d'abord de l'accélération de toutes les circulations, matérielles et humaines, dont l'expression la plus riche est le voyage, lieu de l'acculturation prioritaire des élites et fondement de la construction d'une identité de circulation s'exprimant de façon particulièrement riche dans le cosmopolitisme ${ }^{1}$. Celui aussi, en second lieu, de l'apparition vigoureuse du fait identitaire national et de la rencontre entre la définition particulière de l'appartenance et les procédures de contrôle. La Révolution et son écho européen constituent ici un moment fondamental mais qui ne peut être séparé des pratiques et des pensées observées en aval et en amont. La situation de notre temps, voire l'expérience de chacun incitent également à réfléchir dans une perspective plus longue, sur cette contradiction encore vivante régnant entre l'universalisme et la fermeture. La Révolution technique entamée au $\mathrm{XIX}^{\mathrm{e}}$ siècle avec le chemin de fer, le bateau à vapeur, poursuivi au $\mathrm{XX}^{\mathrm{e}}$ siècle avec la voiture et l'avion ouvre un monde sans limite et sans fin, dont les expériences politiques et culturelles sont fondamentales. De même, la révolution du tourisme, le développement du niveau de vie et des loisirs, l'élargissement de l'espérance de vie et des libertés qui lui sont liées produit l'accélération, sans doute jamais vue dans l'histoire, d'une circulation sans autre finalité qu'ellemême et, du même coup, d'une inégalité nouvelle à l'échelle de la planète puisque $80 \%$ des pays pourvoyeurs de touristes se situent en Amérique du Nord et en Europe occidentale. La visibilité et les nouvelles contraintes induites par ces déplacements fondent une différenciation majeure par rapport aux sociétés anciennes ${ }^{2}$.

\section{MOBILITÉ, VOYAGE, CIRCULATION}

Parler de mobilité plus que de voyages offre une occasion d'élargissement pour notre interrogation. Ainsi il est possible d'éviter de caractériser le voyage ancien seulement par sa valeur et celui d'aujourd'hui par le mépris d'une activité relevant de la marchandise plus que de la culture. D'autres catégories peuvent aussi intervenir que celle de la découverte culturelle définie non sans a priori

1. Pomeau, 1966 ; AtKinson, 1972.

2. Urbain, 1991 ; Boyer, 1972 ; MaC CAnnel, 1976. 
valorisant. Le mouvement des anciens voyageurs, celui des intellectuels, celui des amateurs, rejoint alors la circulation plus générale, celle du commerce des idées, et s'inscrit dans un ensemble plus vaste ${ }^{3}$. Le mot mobile venu du latin et qui apparaît comme adjectif au milieu du XVI ${ }^{\mathrm{e}}$ siècle recouvre un domaine étendu concernant tout ce qui peut changer de place, l'espace, la chronologie, les fêtes mobiles, tout ce qui n'est pas fixe ou fixé, l'ambulant, le nomade, ce qui change, ainsi que les apparences, ce qui est mouvant et instable. Dès lors, le vocable va suivre dans sa trajectoire un questionnement qui le relie à tous les problèmes de la physique, du mouvement du corps, à tous les problèmes de la fortune, des effets et des meubles. Son antithèse définit ce qui reste immobile, et les patrimoines, enracinés. La mobilité elle-même a signifié, toujours à partir du latin, mobilitas, et dès le XIII ${ }^{\mathrm{e}}$ siècle, une valeur sémantique particulière. C'est le caractère de ce qui est mu, de ce qui change, en particulier dans le domaine des passions et de la morale, de l'esprit, de l'agitation d'idées. Le mouvement invite donc à réfléchir à la course, à la trajectoire, à la circulation des objets, des marchandises, des capitaux, aux évolutions et à la capacité de jouer avec l'espace, avec la rapidité ou la lenteur, avec les rythmes du monde et les mécanismes de leur propagation.

Replacer les voyages dans la mobilité, le mobile et les mouvements permet d'y réfléchir autrement que par les catégories de la découverte culturelle mais, de façon plus ouverte, à tous les types des relations à l'espace, à tous les usages et à tous les effets des déplacements. Parler de mobilité permet de déconstruire l'idée qu'il y a un sujet absolu du voyage dans l'histoire et de réfléchir ainsi à la façon dont la culture mieux connue du voyage ne peut se séparer d'un ensemble de mouvements moins connus ou en tout cas moins illustrés par la littérature canonique. L'enjeu est social. Il retrouve autrement une question capitale posée à la culture occidentale dès le Moyen Âge et sans doute avant, développée à l'époque moderne et exacerbée, voire théorisée à l'époque contemporaine ${ }^{4}$, celle de l'opposition entre sédentarité et mobilité, nomades et sédentaires ${ }^{5}$. L'Europe et ses sociétés n'ont eu de cesse de lutter contre l'errance avec une efficacité changeante et suivre le mouvement historique pour fixer, borner, contrôler les hommes, demeure nécessaire à toute philosophie politique, à toute histoire de la Liberté, à toute analyse de la relation de fascination et d'exclusion qui s'impose à l'égard de l'autre et de l'étranger.

3. Nous renvoyons aux principaux dictionnaires de langue et aux grandes encyclopédies : FurEtiÈre, 1690, DidEROT, 1765, ACADÉMIE FRANÇAISE, 1694.

4. BEAUne, 1983.

5. Dagognet, 1984, p. 42-57. 


\section{SÉDENTARITÉ-MOBILITÉ}

Deux valeurs de la civilisation s'affrontent ici. Celle de la Pensée de Pascal : «Tout le malheur des hommes vient d'une seule chose qui est de ne savoir demeurer en repos dans une chambre ${ }^{6}$. » Celle du plaidoyer de Rousseau, dans les Confessions ou dans l'Émile pour justifier l'enrichissement et la nécessité de l'errance pédagogique : «Voyager à pied c'est voyager comme Thalès, Platon, Pythagore. J'ai peine à comprendre comment un philosophe peut se résoudre à voyager autrement et s'arracher à l'examen des richesses qu'il foule aux pieds et que la terre prodigue à sa vue ${ }^{7}$. » Derrière ces deux visions, deux sociétés, deux cultures se distinguent comme des types idéaux, comme des repères distinctifs, où les individus et les groupes sociaux se rencontrent ou s'opposent. La Terre, le royaume paysan, le domaine de la société ancestrale, hiérarchisée avec ses valeurs d'enracinement, de fidélité, de stabilité, dont la principale inscrite au plus profond des mentalités pluriséculaires réside dans le double attachement, symbolique et réel, à la terre, à la richesse foncière et au lieu, composent un premier ensemble. La ville, les ouvertures au monde et à la circulation, l'échange des biens, des hommes, le commerce avec son sens duplice, commerce des idées et mouvement des individus associés dans de multiples réseaux, dans des relations, constituent un second agencement. Dans cette perspective, il ne s'agit pas d'opposer totalement l'un à l'autre domaine, mais plutôt d'en concevoir les rencontres et les imbrications, dans leurs acceptions matérielles ou intellectuelles, dans leurs variables humaines, celle des migrations et du mouvement des masses, celle des circuits qui associent les objets et les hommes. La mobilité et ses cultures peuvent nous aider à comprendre des recoupements qui imprègnent toute la société ancienne et qui peu à peu ont contribué à en bouleverser les équilibres. À mon point de vue, parler de mobilité permet d'adopter sinon un modèle cognitif, du moins l'usage d'un concept, analysable dans le temps et qui travaille comme un canon réflexif les textes les plus variés parce qu'il est à l'œuvre dans la tête des auteurs et des lecteurs, parce qu'il oriente simultanément pratiques, usages, représentations et imaginaires sociaux ${ }^{8}$.

\section{VOYAGEURS ET VOYAGES}

Ce faisant, il faut revenir au voyage élément particulier de ce plus grand ensemble. L'Encyclopédie, et l'auteur de l'article « Voyage », le chevalier de Jaucourt nous y invite avec d'autres auteurs connus, l'ensemble des dictionnaires

\footnotetext{
6. PASCAL, 2000, p. 583.

7. Rousseau, 1969 , p. 826-832.

8. Goldzinc, 2001, p. 5-6.
} 
témoignent ici à travers l'état de la langue des attentes et des idées reçues d'un milieu de lecteurs. Le lexicologue philosophe souligne trois tensions principales : celle qui oppose l'espace étroit, la maison, à la vaste étendue de l'univers, le déplacement mobilise alors de multiples finalités, d'innombrables objets, une grande variété de fonctions ; celle qui oriente une formation et impose la forme canonique du récit de voyage pour véhiculer une instruction, une éducation dont la portée est débattue ; celle enfin, qui voit dans tout voyage une métaphore de la vie : il faut toujours faire le grand voyage. L'idée d'un transport multiple des objets, des idées, des hommes assure l'unité de l'usage à travers une pluralité de fonctions - commerce, éducation, religion, curiosité esthétique, nécessités de la vie ordinaire - et une diversité de situations historiques de l'Antiquité à l'époque des Lumières. Si les grands hommes de l'âge antique sont les meilleurs garants de la pratique, c'est qu'ils découvraient la meilleure école de la vie pour améliorer le corps et l'esprit, pour déchiffrer avec profit le grand livre du monde ou en contempler le théâtre divers.

«Aujourd'hui les voyages dans les états policés de l'Europe (car il ne s'agit point ici de voyages de long cours) sont au jugement des personnes éclairées une partie des plus importantes de l'éducation de la jeunesse et une partie de l'expérience des vieillards » :

ils étendent l'esprit, l'élèvent, l'enrichissent, accroissent les connaissances et guérissent des préjugés nationaux. C'est pourquoi, mais on pourrait reprendre ce thème plus à fond, on n'y supplée pas par les livres et l'occurrence « Voyageur » montre combien il faut se méfier des récits :

«Les voyageurs usent de peu de fidélité ; ils ajoutent presque toujours aux choses qu'ils ont vues, celles qu'ils pouvaient voir ; et pour ne pas laisser le récit de leurs voyages imparfaits, ils rapportent ce qu'ils ont lu dans les auteurs ${ }^{9}$. »

Jaucourt pose alors clairement le problème de la fiabilité du récit ; citant le mot de Strabon : «Tout voyageur est un menteur », reconnaissant à certains auteurs des relations un statut de vérité différent et utile, il ouvre la voie à une interrogation plus générale où il s'agit de comprendre le voyage par rapport à une mobilité plus vaste et à un rôle culturel où l'échange spatial est inséparable de l'échange dans le temps, la personne du voyageur et sa modification inhérentes à la relation comme au déplacement même.

S'il est désormais nécessaire de s'interroger sur les conditions d'une telle expérience, poser la question pourquoi voyage-t-on ? comment voyage-t-on ? et celle de l'importance numérique des voyageurs et celle des modalités du voyage, c'est pour comprendre au-delà de l'acte individualisé et fortement

9. JAUCOURT, 1765, p. 476-478; KANT, 1986, p. 940. 
personnalisé, à quoi correspond la décision qu'il faut prendre pour rompre temporairement ou plus longuement, voire définitivement, le lien qui, dans la société de la stabilité, unit les individus à leur milieu d'origine et donc à euxmêmes. Raison, intelligence, misère, besoins, contraintes ou libertés ont à chaque fois leur mot à dire de même que tout voyage s'insère dans des composantes collectives et que le départ d'un voyageur, migrant ou pèlerin, curieux ou amateur du Grand Tour, n'est pas séparable d'exigences sociales et de réseaux et de règles préexistants à l'aventure. De surcroît, la mobilité et son histoire ne peuvent se comprendre sans référence aux infrastructures qui en conditionnent le déroulement, routes, moyens de transports, lieux d'accueil, institutions de contrôle social, nécessité de connaissance et besoins de régulation ${ }^{10}$. Toute mobilité, tout voyage supposent l'apprentissage de multiples clefs car on ne se déplace jamais n'importe comment dans une errance inorganisée, et la nature des codes peut changer avec celle du déplacement ou avec le niveau social du voyageur, mais leur finalité reste la même, livrer des signes et des moyens qui garantissent le succès et orientent les trajets. Le vêtement du compagnon et la correspondance entre maîtres et ouvriers des villes de production, entre les Cayennes et les gîtes d'accueil, ne sont pas de nature différente par rapport aux outils principaux de la communication des élites voyageuses, une culture des apparences comparables, une organisation apprise des contacts et des lieux à parcourir, un carnet d'adresses implicite ou explicite et des lettres de recommandation font le succès des uns comme celui des autres ${ }^{11}$. Leur absence induit l'échec, leur présence se révèle dans les récits, elle en alourdit la topique quelquefois. C'est dans ce territoire que l'on perçoit comment les normes influent sur la mobilité et celle-ci sur les normes par la dynamique qui touche différents niveaux du territoire : communauté, ville, région, État. C'est ainsi qu'une double tension se fait jour et recompose le tissu social ${ }^{12}$; entre identité, attachement communautaire et différenciation, altérité, entre universalité et communauté des hommes qui définissent mœurs et sociabilité, communs usages et références unitaires, entre valeurs du changement souhaité et conformisme prégnant.

\section{ESPACES ET MOBILITÉS}

Quatre relations anthropologiques classiques caractérisent les cultures de la mobilité : un rapport à l'espace, une conformité au temps, une production sociale et une acculturation pratique tant intellectuelle que matérielle. La circulation

10. Roche, dir., 2000, en part. le texte de Vincent MiLliot.

11. MÉnÉtra, 1982 ; Young, 1976.

12. Je remercie Dominique Margairaz de ses réflexions sur les Territoires, la norme, les mobilités. 
générale que le XVIII ${ }^{\mathrm{e}}$ siècle voit s'accroître suppose et met en valeur une maîtrise de l'espace modifée par rapport aux normes de la société traditionnelle, holiste, inégalitaire et conçue comme immobile dans le temps et fixée dans l'espace de la seigneurie, de la paroisse, de la communauté d'habitants et du finage $^{13}$. Si l'immobilité totale n'existe vraisemblablement pas, son idéal définit le village vertueux et les populations sages par rapport à la confusion des chemins et au désordre de la route. Être sans feu, ni lieu, sans aveu, sans recours ouvre tous les chemins de la méfiance dans le calme des horizons bloqués qui sans être la seule dimension des anciens terroirs en constitue l'asymptote idéalisé. Cependant, les avantages et les vertus du déplacement et de l'échange qu'il autorise ne sont pas méconnus car ils peuvent déboucher sur la transformation et l'enrichissement. Il est porteur d'espoir spirituel et de recours comme on peut le lire dans la société pérégrine ${ }^{14}$, comme dans le commerce il peut conduire à la fortune et à la mobilité sociale. C'est pourquoi il faut désormais relire avec attention ces phénomènes massifs qui jettent sur les routes pèlerins, travailleurs, hommes de négoce et d'affaires aux rayons d'actions diversement tracés. Alphonse Dupront nous l'a montré, le pèlerinage est une marche vers un ailleurs, et le pèlerin est d'abord un homme qui marche pour devenir autre comme le prouvera au retour son surnom, le Jacquet qui a fait le Tour jusqu'à Saint-Jacques-de-Compostelle, le Roumieu qui a atteint Rome ${ }^{15}$. Si, sur les grands chemins, pèlerins et marcheurs de Dieu se confondent avec la foule imprécise des errants qu'on surveille avec de plus en plus d'attention, leur avance et leur retour n'est pas une errance, ils ont un but, ils ont une progression cathartique, ils s'inscrivent dans une organisation. D'ailleurs, même en ce qui concerne les pauvres et les marginaux le hasard ne règne jamais seul sur leurs circulations que peuvent infléchir les circonstances mais qui dépendent également de démarches solidement installées et construites par l'habitude inséparables et inséparées des pratiques de la migration temporaire et des institutions hospitalières ${ }^{16}$. Des sentiers invisibles conduisent depuis longtemps pauvres et moins dépendants et la position socio-économique ne suffit pas à expliquer la mobilité et son déroulement. Comme pour le pèlerin, mais peut-être avec moins de certitude, le lieu à atteindre s'identifie à une promesse, la peine pour y arriver admise par tous compte comme une avance sur la récompense attendue. L'espoir du retour n'est jamais anéanti malgré les difficultés.

C'est pourquoi, il faut être attentif à l'imaginaire qui est à l'œuvre dans la rupture, et aux rapports qu'il entretient avec le réel. Le roman des Lumières est porteur de cette liaison entre mobilité sociale et circulation des hommes.

13. BOUCHARD, 1972 ; LEMAÎTRE, 1978.

14. Julia et Boutry, 2000.

15. Dupront, 1967 et 1987.

16. Châtelain, 1967 ; Fontaine, 1984, p. 27-44, 78-93 et 113-140. 
L'attraction urbaine en est le moteur principal comme l'a souligné Jean-Claude Perrot, et, toutes les œuvres littéraires majeures

« renvoient l'image de ce rêve urbain si prégnant, dans son parfum de fruit défendu, et trois miroirs de la société jalonnent le chemin parcouru par les Français dans la tentation comme aussitôt dans le regret, Marivaux vers 1740, Rousseau vers 1760, Restif de La Bretonne dans les années $1780^{17}$ ».

La condamnation de la déculturation urbaine par les élites savantes et morales n'est certainement que la vision inversée des espérances populaires et le phénomène intègre plusieurs dimensions. Le déplacement peut être la condition et l'occasion d'une transformation positive, c'est une obligation du métier, c'est une nécessité de la tradition professionnelle ou régionale. C'est parfois une cause de blocage, dans une carrière, dans un niveau social, dans une sociabilité comme peuvent le vivre les individus contraints au départ, à la fuite, ainsi dans la migration du refuge huguenot ou dans l'émigration des temps révolutionnaires, mais tant au niveau individuel qu'au plan collectif toute la question est de savoir ce que l'on peut oublier ou ce que l'on peut apprendre, pour paraphraser la célèbre formule de la Restauration. À l'œuvre dans tous ces déplacements on retrouve un apprentissage où, quels que soient le niveau social et la culture, la transmission de l'information se fait par l'oral et par l'écrit, par l'imprimé de grande diffusion, le guide populaire ou savant, comme par la relation entendue à l'auberge ou lue dans la version lettrée ou l'extrait résumé du journal. De même, les pratiques de tous sont balisées et intériorisées, une grille de topoï oriente la représentation spatiale et l'attente. Enfin, la dimension imaginaire et sensible guide les curiosités qui se déplacent d'un milieu à l'autre dans le temps, comme la perception des risques et des dangers, celles des merveilles et des mondes exotiques et étranges du fait même de leur éloignement. L'avocat Charles Georges Thomas Garnier s'en fait l'écho dans l'avertissement qui ouvre le premier volume de la collection de Voyageurs imaginaires, songes et visions, romans cabalistiques, 40 volumes, in- $8^{\circ}$, publiés à 3 livres 12 sols au rythme de deux tous les mois, comme l'annonce Le Mercure de France le 28 juillet en $1787^{18}$ :

«Le philosophe sans autre guide que son imagination se transporte dans des mondes nouveaux, où il recueille des observations qui ne sont ni moins intéressantes ni moins précieuses. Suivons le dans ces courses et soyons assurés de rapporter autant de fruit de nos voyages que si nous avions fait le tour du monde. »

17. PERROT, 1975, vol. I, p. 158-159.

18. Goulemot, 1990, vol. II, p. 85-97. 
La valorisation de la fiction s'affirme en parallèle à la vogue des récits de voyages réels et elle entretient une relation entre le déplacement imaginaire et romanesque guidé par le goût de l'évasion, de l'émotion, du sensible, l'attrait pour la quête philosophique et l'intérêt pour les mœurs ou la compréhension exotique. Les jeux d'espace sont en présence dans les choix du réel comme dans ceux de l'écriture ${ }^{19}$.

\section{CIRCULATION MAÎTRISE DU TEMPS}

L'histoire du temps comme celle des moyens de sa mesure ont mis en évidence la part du rapport à l'espace ${ }^{20}$. Partir, vivre hors du local, traverser les échelons imbriqués de la distanciation spatiale révèle une capacité à inscrire son existence dans un hors temps et en même temps crée des besoins afin de garder un accès à plusieurs temporalités dont les plus prégnantes agissent comme deux pôles magnétiques entre l'enracinement du village et le bouillonnement actif de la ville. Le déplacement est déjà rupture avec les rythmes du quotidien, les séquences de la nécessité villageoise des cycles naturels, des fêtes liturgiques et coutumières, du temps compté et cependant faiblement mesuré à l'aune des saisons et de la succession des jours. Le voyage crée désadaptation et réadaptation à de nouveaux partages liés souvent au travail, à l'échange proche ou lointain. Toutefois, c'est aussi une communication entre le présent et l'avenir, porteuse de formation et de projets d'installation et de retour. Le mariage et l'établissement de l'ouvrier ou du colporteur migrant peuvent être à ce prix mais la réalisation peut être à plus ou moins long terme et le rêve du pays natal se dissoudre dans une intégration nouvelle. L'ensemble temporel de la mobilité au même titre que son organisation spatiale implique un jeu de temporalité. Celui de la répartition, saisonnière ou plus resserrée, celui de l'indétermination, qui fait dépendre le trajet du hasard ou des occasions offertes, arrêtent la course du pèlerin, orientent celle des compagnons diversement, d'étape en étape, dans une expérience qui ne se répétera jamais et qui par ce caractère unique entraîne parfois à l'aventure. L'itinéraire peut être fixé, le temps mis à le parcourir peut dépendre des choix ou des nécessités. On ne va qu'une fois à Jérusalem, même quand on est Chateaubriand. Enfin, le voyage et ses expériences multiples débouchent sur le temps de la mémoire qui est souvent celui du récit que l'on fait pour les autres restés à l'abri, pour la famille, pour le milieu, son édification et sa transformation. Thomas et Felix Platter en témoignent pour leur cercle bâlois, mais aussi Jacques Louis Menétra ou Louis Simon pour les compagnons ouvriers du XVIII siècle, Agricol Perdiguier ou

19. MARIN, 1973

20. Pionnier et toujours fondamental, Cipolla, 1976 ; Landes, 1983 ; EKEland, 1984. 
Martin Nadaud pour les travailleurs du XIX ${ }^{\mathrm{e}}$ siècle. La littérature de voyage va s'inscrire dans ces jeux d'un temps autre mais plus riche ou présenté comme tel, d'un espace différent propice au changement ou supposé tel, elle y trouvera sa justification principale et sa fascination continuée. «Curiosité n'est que Vanité », dit Pascal, et il ajoute :

« Le plus souvent on ne veut savoir que pour en parler ; autrement on ne voyagerait pas sur la mer pour ne jamais rien dire et pour le seul plaisir de voir sans espérance d'en jamais communiquer ${ }^{21}$. »

Le voir est la médiation du dire, en racontant son voyage tout voyageur le mène à son terme naturel. La conquête du temps permet de donner aux voyages et à leur récit leurs sens, mais aussi d'accroître le savoir et d'accélérer la communication ; le substitut de la vision directe ne fait que prolonger la temporalité d'une expression vécue et proposée à l'exemple. Elle débouche sur l'intemporel. Quand se réduit le temps du voyage, son étendue s'accroît par un plus grand nombre et d'autres conventions descriptives vont remplacer les anciennes.

\section{LES SOCIÉTÉS DU VOYAGE}

De la même manière, les espaces et le temps de la circulation des hommes vont favoriser la création d'un entre-deux social. Que l'on saisisse le voyage dans ses flux collectifs ou dans ses mouvements individualisés, on y voit se constituer des sociétés spécifiques, elles sont temporaires, se nouant et se dénouant selon les étapes du mouvement, ses buts et ses finalités, elles s'organisent selon sa nature. Le modèle est celui du Stage-coach de La Chevauchée fantastique de John Ford illustré avant lui par Flaubert, Maupassant ou plus en amont encore par les romanciers du voyage ou par les auteurs de récits; Montaigne en fournit un exemple mémorable au temps des périples à cheval. Trois caractères s'y découvrent : c'est une société éphémère et instable, elle n'est pas faite pour durer, elle est propice alors aux aventures courtes et aux rencontres ; c'est aussi une société moins sensible aux conventions et aux hiérarchies de la vie ordinaire car on y est pris par ce que l'on souhaite paraître plus que pour ce que l'on est réellement. Le masque et les apparences y règnent troublant les rapports sociaux sans les bouleverser. C'est pourquoi les aventuriers de tout poil ont été des gestionnaires de la mobilité, utilisant les possibilités offertes pour changer sinon de personnalité du moins de qualité sociale. La vie peut alors devenir fiction et celle-ci la vie même, comme le montre le destin de

21. MesnaRd, 1986, p. 10-11. 
Casanova ou de tant d'autres chevaliers de fortune au siècle des Lumières ${ }^{22}$. C'est que la notion d'aventure est propice à expliciter des traits communs à toute mobilité, la rupture avec la routine et l'ennui, l'aspiration à l'inconnu, au changement, à un futur dont la portée sociale est incontestable ${ }^{23}$, car c'est la trame globale de l'existence qui est modifiée. Dans les sociétés anciennes régies par les coutumes, réglées par la connaissance de proximité et le regard des hommes et des femmes, les sociétés urbaines traditionnelles n'échappent pas totalement à cette expérience des autres ${ }^{24}$, le départ, l'absence libèrent des protections habituelles, ouvrent à l'insécurité, favorisent les écarts fussent-ils temporaires, ce faisant ils contribuent à façonner les nouveaux harmoniques de l'individualisme moderne.

C'est pourquoi, il est nécessaire de s'attacher à l'analyse de ces relations fragiles et souvent sans lendemain, à leur articulation sur la rencontre des milieux et des classes, qui confère à la sociabilité une part croissante dans la définition de l'espace public. Dans l'histoire de la mobilité, la notion est essentielle car, à tous ses niveaux, elle éclaire le parcours des voyageurs de toute nature, elle marque les enjeux et les limites de l'accueil et de l'hospitalité et ce qu'autorisent réellement les libertés ouvertes par le brouillage des positions possiblement offert par le déplacement. La solidité des liens noués entre les voyageurs et les manières dont ces relations sont entretenues et poursuivies après le retour constituent l'un des tests par lesquels on peut mesurer la capacité à intégrer l'autre dans un réseau de connaissance élargie, dans une aire de relation que l'on maintient gracieusement à sa disposition ${ }^{25}$. Les sociétés de l'accueil ou de la méfiance coexistent à tous les niveaux sociaux et nourrissent leurs imaginaires de fantasmes multiples, la fascination valorisante et le danger irraisonné font la culture des sociabilités mobiles. Le passage de l'hospitalité, vertu antique et gratuite, à l'économie de l'hospitalité marque alors une étape. L'auberge et l'hôtel ne naissent pas du voyage de l'élite curieuse mais du mouvement massif et généralisé, temporaire ou plus durable des hommes. Dans un mouvement qui n'est pas linéaire et qui ne se développe pas partout aux mêmes rythmes, la fonction touristique est un élément qui s'ajoute à d'autres antérieurs et qui font de l'auberge ancienne soit un lieu fortement ancré dans la vie des populations locales - c'est le cas des zones riches et densément peuplées de l'Europe du XVI et du XVII ${ }^{\mathrm{e}}$ siècle -, soit une institution rare, et en tout cas réservée aux étrangers - c'est le cas des régions moins peuplées et moins développées - qui est directement liée à l'infrastructure routière et aux liaisons entre les villes, aux marges orientales ou méridionales de l'Europe ${ }^{26}$.

22. Roth, 1980 ; StoEv, 1997.

23. SIMMEL, 1989 , p. 304-325.

24. FARGE, éd., 1979 ; FARGE, 1986.

25. BirnBAuM, 1997 , p. 39-50.

26. MAÇZAK, 1995, p. 65-68. 
C'est pourquoi leur confort, diversement mesuré aux niveaux des habitudes des voyageurs, reste un test de la différenciation économique et un révélateur des attentes. Entre l'offre et la demande, une étude de l'économie de l'accueil, par exemple à Paris, entre le $\mathrm{XVI}^{\mathrm{e}}$ et le $\mathrm{XIX}^{\mathrm{e}}$ siècle, montre comment elle est inséparable d'une acculturation dans le domaine de la culture matérielle et de la civilité 27 .

\section{L'ACCULTURATION ENJEU DE LA MOBILITÉ}

Le postulat de toutes les études consacrées au voyage c'est qu'il est destiné à transformer les hommes, et qu'il serait sans intérêt s'il n'était expérience et enseignement. Métaphore de la vie humaine, il doit conduire à des réalités plus essentielles $^{28}$. La transformation des individus est implicite, mais on peut se demander si elle a été mise en œuvre et réelle pour tous au même titre. Le comment, quand le vu donne vie au lu, les manières acquises par contact et par imitation, sont bien perçus dans les récits de voyage et leur succès éditorial qui ne cesse de croître du XVI ${ }^{\mathrm{e}}$ au XIX ${ }^{\mathrm{e}}$ siècle parle en ce sens ${ }^{29}$. Leur défaut est de masquer les résistances car, comme tous les moyens de la communication, ils servent à alimenter les conversations, à transformer la réalité et les idées en opinion, à transmettre moins ce qui est que ce qui doit être vu. Leur témoignage est fragile mais particulièrement précieux car il livre le cheminement de la mémoire et des souvenirs dans leur mise en forme et jusqu'à leur transmission. Toutefois, ce mouvement du discours sur le monde ne coïncide qu'en partie avec le mouvement réel et s'en tenir à son analyse seule identifie le récit de l'expérience avec sa transcription codifiée. Pour la république des lettres, pour la naissance du tourisme, ils construisent une conscience commune moins par la réalité d'une pratique que par sa lecture. On en retiendra l'intérêt pour définir des mobilités essentielles dans la vie culturelle du petit monde des lettrés et des aisés et on pourra y découvrir les traces et les indices trop rares de contraintes qui s'imposent largement à tous. Le genre intervient ici, au même titre que la fiction, ou que la littérature apodémique. C'est un moyen de découvrir la transformation des voyageurs et la topique qui procède à la présentation de ce cheminement vers soi à travers l'espace du déplacement. Elle mobilise des lieux, des monuments, des thèmes, elle vise à créer des effets de vérité à travers l'ordonnancement du récit. Mais, si l'on veut comprendre au-delà du voyage cultivé, culturel, les effets de la mobilité, il faut le confronter à d'autres catégories interprétatives.

27. Roche, dir., 2000, p. 291-352.

28. Mesnard, 1986, p. 10-11.

29. Roche, 1978, vol. I, p. 320-322 et vol. II, p. 510-513. 


\section{LUMIÈRES ET MOBILITÉS}

Le $\mathrm{XVIII}^{\mathrm{e}}$ siècle exalte la figure du voyageur, symbole héroïque de la conquête des mondes lointains, héros d'une expansion et de l'universalisation des valeurs de l'Europe : Cook raconté par Forster, Bougainville complété par Diderot, en illustrent les forces et les faiblesses ${ }^{30}$. De fait, la gloire des découvreurs et des pionniers ne se pare pas seulement de cette affirmation d'une volonté d'assujettissement générale de l'univers, celle-ci peut intellectuellement commencer à la porte de chacun et en tout cas au-delà des frontières diversement vécues alors de chaque État. Les voyageurs et leurs récits développent une conception nouvelle de la raison opérante et celle-ci se dévoile et se confirme à des degrés divers dans l'ensemble des sociétés, les petites gens n'y échappent pas qui veulent aussi connaître et s'approprier des leçons apprises ailleurs. C'est pourquoi plutôt que de reprendre une typologie toujours difficile à caractériser car on ne sait pas quelle en serait la théorie analytique, on peut comprendre la mobilité comme le résultat de ce qu'imposent la coutume, les habitudes ou l'habitus, l'imposition de plusieurs exigences intériorisées et les choix des individus toujours négociées par des relations de convention. Le proche et le lointain sont deux premières catégories dont la tension apparaît dans de multiples développements, ils organisent le rapport des villageois à des espaces, à des aires naturelles et sociales où l'intérêt du déplacement compense le coût, économique et psychologique. On peut en mesurer l'effet dans l'établissement des réseaux matrimoniaux, dans la fréquentation mesurée de l'aire des marchés et des foires ${ }^{31}$, dans les circuits du négoce mais également dans des pratiques professionnelles intellectualisées, celles de l'enquête administrative ou de l'évaluation des moyens productifs. À la fin du XVIII ${ }^{\mathrm{e}}$ siècle, le comte Léopold Berchtold proposera à tous les voyageurs ce modèle éclairé comme un moyen de comprendre le monde et d'utiliser les leçons du voyage pour assurer l'avenir de la patrie originelle. Quand Louis Simon quitte son village pour un bref tour dans la France du Nord-Ouest, il mêle sa curiosité professionnelle et technique à son goût pour la découverte des villes inconnues et de leurs monuments, à sa manière de vivre avec une expérience religieuse. La distance, la rupture temporaire sont alors gages d'un enrichissement gagné plus par choix libre que par obligation ${ }^{32}$.

Les contraintes et les libertés contribuent à créer les dispositifs incitatifs aux départs et organisant la maîtrise du temps et de l'espace. L'obligation professionnelle s'impose plus ou moins fortement, dans nombre de métiers, c'est une étape nécessaire à la formation, et la mobilité pédagogique ne se lit

30. Bourguet, 1996 ; Hersant, 1995.

31. Margairaz, 1988 ; Perrot, 1975, vol. I, p. 467-494.

32. Fillon, 1996 ; VAsSort, 1999. 
pas seulement dans les variantes sociales de la peregrinatio academica. L'administration des âmes, celle des corps et des choses sont tout autant incitatives que les nécessités d'un apprentissage social, d'une pédagogie artistique, d'une propédeutique à l'établissement dans lesquelles on enferme les voyageurs. La formation, l'agrément, la connaissance des autres font la connaissance de soimême. On entend dans la nécessité des auteurs de récit de voyage d'avoir à rapporter des faits normés et imposés cette principale tension du genre, où progressivement s'impose le triomphe du moi dont Stendhal sera sans doute le plus illustre défenseur. L'agrément mondain, la joie des sociabilités itinérantes des peuples sur les routes vont dans le même sens, qu'illustre, dès le XVI ${ }^{\mathrm{e}}$ siècle, l'adage « Ce que le cœur ne voit le cœur ne décrit » et que confirme définitivement la proclamation d'Henri Beyle : « Je ne voyage pas pour connaître l'Italie mais pour me faire plaisir. »

Le contraste entre sédentaires et nomades migrants et enracinés se nuance alors de multiples facettes. La société des voyageurs n'est jamais totalement séparée de celle sans doute plus nombreuse de ceux qui restent, mais elle a sa démographie et ses traits propres et c'est dans la façon dont ceux-ci interrogent d'une manière croissante toutes les formes d'autorité que s'est jouée l'histoire des contrôles de population, celle de la régulation réussie ou inachevée des circulations, celle de la définition des étrangers donc de l'identité nationale.

Au total, resserrer les liens qui existent entre la mobilité, la circulation, les voyages infléchit une histoire qui a été centrée sur la médiation des idées et des mœurs, voire celle des habitudes matérielles vers un ensemble d'expériences où s'élabore la signification socioculturelle d'une rupture : entre un temps imaginé comme celui de la stabilité et un temps où tout s'accélère. La confrontation des usages sociaux et des représentations qui les construisent montre une fois encore que l'on ne peut confondre le voyage et son récit, pas plus que la carte et le territoire. Privilégier le déplacement dans le cadre des sociétés traditionnelles et ses acteurs multiples permet seul de comprendre le lien qui s'établit de façon spécifique entre une situation banale souvent, exceptionnelle parfois, et ceux qui la vivent. De nouveaux moyens techniques, des dynamismes plus puissants changent la donne au XIX ${ }^{\mathrm{e}}$ siècle. La rupture pare encore des lueurs de la nostalgie et de l'idéalisation ce qui était le temps supposé d'un tourisme authentique mais qui justement naît dans l'inconfort et l'incertitude des déplacements anciens comme l'a montré Marc Boyer ${ }^{33}$. La rupture que l'on peut lire dans les autres formes de gestion du temps et de l'espace nées avec l'accélération de la vie et la vitesse grandissante introduit des transformations fondamentales : l'isolement remplacé par la proximité à une échelle impensable jusqu'alors, le rapport à ce que l'on voit et l'intensification de la vie nerveuse, la modification des rapports sociaux élaborés dans l'artificialité des conditions

33. BOYER, 1999. 
nouvelles du voyage et du loisir. Et, pour le dire en d'autres termes, quel est le lien réel qui s'est tissé dans l'expérience sensible, matérielle et intellectuelle de la mobilité, entre le progrès et la circulation ? L'ouverture des sociétés traditionnelles ne s'est vraisemblablement pas faite de façon linéaire ${ }^{34}$, elle a pu connaître ses avancées et ses reculs mais le problème historique qu'elle pose est bien celui de la manière dont se constitue une culture du changement par le refus du repliement sur soi, du repli sur le passé, le lieu ${ }^{35}$.

Daniel Roche (octobre 2000).

34. Croix, 1999.

35. Koselleck, 1990, p. 31-35 et 263-296. 


\section{LISTE DES RÉFÉRENCES}

AtKinson (Geoffroy), 1972, Les Relations de voyage et l'évolution des idées. Contribution à l'étude de la formation de l'esprit du XVII' siècle, $1^{\text {re }}$ éd. 1927, ici Genève, Slatkine.

BeAunE (Jean-Claude), 1983, Le Vagabond et la machine. Essai sur l'automatisme ambulatoire. Médecine, technique et société, 1880-1910, Seyssel, Champ Vallon.

Birnbaum (Jean), 1997, Arthur Young en Europe. Culture du voyage et cheminement des Lumières au XVIII siècle, mémoire de maîtrise, université de Paris I.

Bouchard (Gérard), 1972, Le Village immobile. Sennely-en-Sologne au XVIII e siècle, Paris, Plon.

Bourguet (Marie-Noëlle), 1996, «L'Explorateur», in Vovelle (Michel), dir., L'Homme des Lumières, Paris, Seuil, p. 286-346.

BOYER (Marc), 1972, Le Tourisme, Paris, Seuil.

Boyer (M.), 1999, Histoire du tourisme de masse, Paris, Presses universitaires de France (Que sais-je ?, 3480).

ChÂtelain (Abel), 1967, «Les migrations temporaires au XIX ${ }^{\mathrm{e}}$ siècle », Annales de démographie historique, p. 9-28.

Cipolla (Carlo M.), 1976, Clocks and culture, 1300-1700, New York-Londres, W. W. Norton and Co.

Crorx (Alain), 1999, «L'ouverture des villages sur l'extérieur fut un fait éclatant dans l'ancienne France », Histoire et sociétés rurales, 11, p. 109-146.

Dagognet (François), 1984, Le Nombre et le lieu, Paris, Vrin.

Dupront (Alphonse), 1967, « Tourisme et pèlerinage », Communications, p. 97-121.

Dupront (A.), 1987, Du sacré. Croisades et pèlerinages, images et langages, Paris, Gallimard.

EKElAND (Ivar), 1984, Le Calcul et l'imprévu. Les figures du temps de Kepler à Thom, Paris, Seuil.

FARge (Arlette), éd., 1979, Vivre dans la rue à Paris au XVIII siècle, Paris, GallimardJulliard.

FARGe (A.), 1986, La Vie fragile. Violence, pouvoirs et solidarités à Paris au XVIII siècle, Paris, Hachette.

Fillon (Anne), 1996, Louis Simon, villageois de l'ancienne France, préf. de Pierre Chaunu, Rennes, Éditions Ouest-France.

Fontaine (Laurence), 1984, Le Voyage et la mémoire. Colporteurs de l'Oisans au $X I X^{e}$ siècle, Lyon, Presses universitaires de Lyon.

Goldzink (Jean), 2001, Montesquieu et les passions, Paris, Presses universitaires de France.

Goulemot (Jean-Marie), 1990, « Nouveautés. Les utopies », in Chartier (Roger) et Martin (Henri-Jean), dir., Histoire de l'édition française. Vol. II : Le Livre triomphant, Paris, Fayard-Cercle de la librairie. 
Hersant (Yves), 1995, « Sur les routes d'Europe », in Roger (Philippe), L'Homme des Lumières, de Paris à Petersbourg, Naples, Vivarium, p. 65-78.

JAUCOURT (Louis, chevalier de), 1765, in Encyclopédie ou Dictionnaire raisonné des sciences, des arts et des métiers, par une Société de gens de lettres, mis en ordre et publié par Denis DideroT... et quant à la partie mathématique par M. D'AlEMBERT, vol. XVII, Neuchâtel, S. Faulche.

Julia (Dominique) et Boutry (Philippe), 2000, Pèlerins et pèlerinages dans l'Europe moderne, Rome, École française de Rome.

KaNT (Emmanuel), 1986, Anthropologie du point de vue pragmatique, in Euvres. Vol. III, Paris, Gallimard (Bibliothèque de la Pléiade).

Koselleck (Reinhart), 1990, Le Futur passé. Contribution à la sémantique des temps historiques, trad. de l'allemand Jochen Hoock et Marie-Claire Hoock, Paris, Éditions de l'École des hautes études en sciences sociales.

LANDES (David Saul), 1983, Revolution in time. Clocks and the making of the modern world, Cambridge, MA, Belknap Press of Harvard University Press.

LEMAîTRE (Nicole), 1978, Un horizon bloqué. Ussel et la montagne limousine aux XVII et XVIII siècles, Ussel, Musée du pays d'Ussel.

Les Récits de voyage, 1986, éd. Centre d'étude et recherche d'histoire des idées et de la sensibilité, préf. Jean Mesnard, Paris, Nizet.

Mac Cannel (John), 1976, The Tourist. A new theory of the leisure class, New York, Schocken books.

MAÇZAK (Antoni), 1995, Travel in early modern Europe, trad. angl. Ursula PHILLIPS, Cambridge, Polity Press.

Margairaz (Dominique), 1988, Foires et marchés dans la France préindustrielle, Paris, Éditions de l'École des hautes études en sciences sociales.

MARIN (Louis), 1973, Utopiques. Jeux d'espaces, Paris, Minuit.

MÉNÉTRA (Jacques-Louis), 1982, Journal de ma vie (1738-1812), éd. Daniel Roche, Paris, Montalba.

MESNARD (Jean), 1986, préf., in Les Récits de voyage, op. cit. supra.

Pascal (Blaise), 2000, Euvres complètes. T. II. Paris, Gallimard (Bibliothèque de la Pléiade).

Perrot (Jean-Claude), 1975, Genèse d'une ville moderne. Caen au XVII' siècle, La Haye, Mouton (École des hautes études en sciences sociales, Centre de recherches historiques), 2 vol.

Pomeau (René), 1966, L'Europe des Lumières. Cosmopolitisme et unité européenne au dix-huitième siècle, Paris, Stock.

Roche (Daniel), 1978, Le Siècle des Lumières en province. Académies et académiciens provinciaux, 1660-1789, Paris/La Haye, EHESS/Mouton, 2 vol.

Roche (D.), dir., 2000, La Ville promise. Mobilité et accueil à Paris (fin XVII -début XIX ${ }^{e}$ siècle), Paris, Fayard, en part. Vincent MiLLIOT, « La surveillance des migrants et les lieux d'accueil à Paris du XVI ${ }^{\mathrm{e}}$ siècle aux années 1830 », p. 21-76, et Daniel Roche, «Logeurs et hôteliers. Dépenses, consommations, sociabilités », p. 291-352.

Roth (Suzanne),1980, Aventure et aventuriers au XVIII ${ }^{e}$ siècle. Un essai de sociologie littéraire, Lille, Atelier de reproduction des thèses de l'université de Lille 3, 2 vol.

Rousseau (Jean-Jacques), 1969, Euvres complètes. T. IV : Émile, éducation, morale, botanique, Paris, Gallimard (Bibliothèque de la Pléiade). 
Simmel (Georg), 1989, Philosophie de la modernité. La femme, la ville, l'individualisme, introd. et trad. de l'allemand par Jean-Louis VIEILLARD-BAron, Paris, Payot.

Stoev (Alexandre), 1997, Les Aventuriers des Lumières, Paris, Presses universitaires de France.

Urbain (Jean-Didier), 1991, L'Idiot du voyage. Histoires de touristes, Paris, Plon.

VAssort (Jean), 1999, Les Papiers d'un laboureur au siècle des Lumières. Pierre Bordier, une culture paysanne, préf. de Daniel Roche, Seyssel, Champ Vallon.

Young (Arthur), 1976, Voyages en France, trad. Henri SÉE, Paris, Colin, 3 vol. 